\title{
Lateral epitaxial growth of germanium on silicon oxide
}

V. D. Cammilleri, V. Yam, F. Fossard, C. Renard, D. Bouchier, P. F. Fazzini, L. Ortolani, F. Houdellier, and M. Hÿtch

Citation: Appl. Phys. Lett. 93, 043110 (2008); doi: 10.1063/1.2963363

View online: https://doi.org/10.1063/1.2963363

View Table of Contents: http://aip.scitation.org/toc/apl/93/4

Published by the American Institute of Physics

\section{Articles you may be interested in}

High hole mobility tin-doped polycrystalline germanium layers formed on insulating substrates by lowtemperature solid-phase crystallization

Applied Physics Letters 107, 022103 (2015); 10.1063/1.4926507

Selective growth of $\mathrm{Ge}$ on $\mathrm{Si}(100)$ through vias of $\mathrm{SiO}_{2}$ nanotemplate using solid source molecular beam epitaxy

Applied Physics Letters 83, 5032 (2003); 10.1063/1.1632037

Origin and removal of stacking faults in $\mathrm{Ge}$ islands nucleated on $\mathrm{Si}$ within nanoscale openings in $\mathrm{SiO}_{2}$ Journal of Applied Physics 110, 073516 (2011); 10.1063/1.3643003

Defects reduction of Ge epitaxial film in a germanium-on-insulator wafer by annealing in oxygen ambient APL Materials 3, 016102 (2015); 10.1063/1.4905487

High quality relaxed germanium layers grown on (110) and (111) silicon substrates with reduced stacking fault formation

Journal of Applied Physics 114, 154306 (2013); 10.1063/1.4825130

Electrical properties of pseudo-single-crystalline germanium thin-film-transistors fabricated on glass substrates

Applied Physics Letters 107, 142102 (2015); 10.1063/1.4932376

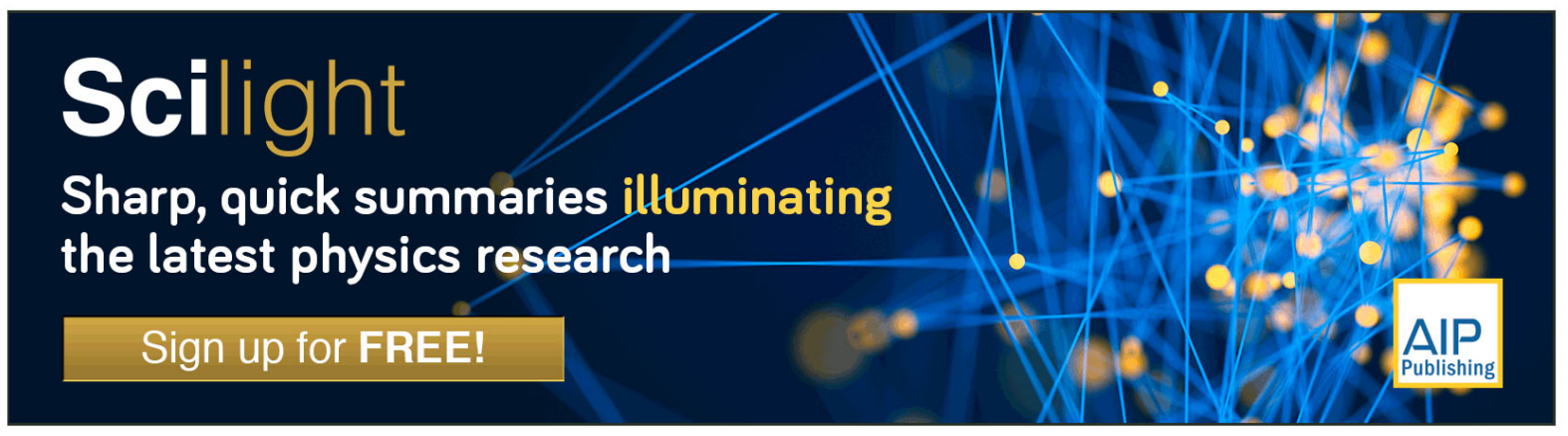




\title{
Lateral epitaxial growth of germanium on silicon oxide
}

\author{
V. D. Cammilleri, ${ }^{1}$ V. Yam, ${ }^{1, a}$ F. Fossard,${ }^{1}$ C. Renard, ${ }^{1}$ D. Bouchier, ${ }^{1}$ P. F. Fazzini, ${ }^{2}$ \\ L. Ortolani, ${ }^{2,3}$ F. Houdellier, ${ }^{2}$ and M. Hÿtch ${ }^{2}$ \\ ${ }^{1}$ Institut d'Electronique Fondamentale, CNRS UMR 8622, Université Paris-Sud, Bât 220, \\ 91405 Orsay, France \\ ${ }^{2}$ CEMES-CNRS, University of Toulouse, 29, Rue Jeanne Marvig, 31055, Toulouse Cedex 4, France \\ ${ }^{3}$ University of Bologna, Department of Physics, v.le B. Pichat, 6/2, 40127 Bologna, Italy and CNR-IMM \\ Bologna, v. Gobetti, 101, 40129 Bologna, Italy
}

(Received 20 May 2008; accepted 3 July 2008; published online 29 July 2008)

\begin{abstract}
We have developed a method using local oxidation on silicon to create nanoscale silicon seeds for the lateral epitaxial overgrowth of germanium on silicon oxide. The germanium growth starts selectively from silicon seed lines, proceeds by wetting the $\mathrm{SiO}_{2}$ layer and coalesces without formation of grain boundary. Analysis by high resolution transmission electron microscopy have shown that Ge layers grown above silicon oxide are perfectly monocrystalline and are free of defect. The only detected defects are situated at the $\mathrm{Ge} / \mathrm{Si}$ interface. Geometrical phase analyses of the microscopy images have shown that the Ge layer is fully relaxed and homogeneous. (c) 2008 American Institute of Physics. [DOI: 10.1063/1.2963363]
\end{abstract}

Germanium used as a high mobility channel, presents a strong interest for fully depleted metal oxide semiconductor field effect transistors to be developed in the next complementary metal oxide semiconductor technology generation $\left(22 \mathrm{~nm}\right.$ and below). ${ }^{1}$ Currently germanium on insulator(GeOI) structures are fabricated in the form of plain wafers by bonding techniques such as etch back or SMARTCUT, ${ }^{2}$ or by $\mathrm{Ge}$ condensation. ${ }^{3}$ In this paper, we propose the lateral epitaxial growth (LEG) of germanium on a $\mathrm{SiO}_{2}$ layer formed by oxidation of a standard silicon wafer as an alternative to these techniques. ${ }^{4}$ Such a local approach have the advantage of enabling easily the manufacturing of $\mathrm{Ge}-$ and Si-based devices at the same level on the wafer, and, from the material point of view, can be effective for defect density reduction, thanks to the low dimensions of the LEG crystals. ${ }^{5}$

It is well known that the main difficulty for growing germanium on silicon stems from a mismatch of $4.2 \%$ between the two crystal lattices, what results, on plain wafers, in the emission of misfit dislocations as soon as the growing film exceeds a critical thickness standing in the order of $1 \mathrm{~nm} .{ }^{6}$ When starting from seeds of limited diameter, a growing mismatched crystal is free of accommodating its lattice in a three-dimensional volume, which opens the possibility of dislocation-free growth. Previous calculations ${ }^{7}$ have shown that for a given mismatch, the critical film thickness strongly depends on the seed radius and tends to the infinite below a critical radius value, which would be close to $20 \mathrm{~nm}$ for a mismatch of $4.2 \%$. The validity of this approach has been demonstrated with the growth of dislocation-free $\mathrm{Ge}$ on silicon covered with a very thin $\mathrm{SiO}_{2}$ film. ${ }^{8,9}$

We have developed a method to create nanoscale silicon seeds for lateral epitaxial of $\mathrm{Ge}$ on silicon oxide by using local oxidation of silicon (LOCOS) within a silicon nitride pattern mask. To realize the LOCOS, a silicon nitride film ( $\sim 50 \mathrm{~nm}$ thick) is deposited on $p$-type $\mathrm{Si}(001)$ substrates by plasma enhanced chemical vapor deposition (PECVD) and patterned by conventional deep UV lithography (248 nm)

\footnotetext{
a) Author to whom correspondence should be addressed. Electronic mail: vy.yam@u-psud.fr.
}

and reactive ion etching. Silicon nitride patterns consisting of $0.3-10 \mu \mathrm{m}$ wide lines oriented along the $\langle 110\rangle$ direction are made. A wet oxidation at $1050{ }^{\circ} \mathrm{C}$ is then performed in order to grow a $\sim 100 \mathrm{~nm}$ thick silicon oxide layer through the nitride mask. At this oxidation temperature, flat $\{111\}$ interfaces between silicon oxide and $\mathrm{Si}$ are formed close to the bird's beaks, as shown in Ref. 10. The oxide layer is then partially etched using a hydrofluoric (HF) acid solution to partially free the $\{111\}$ facets from $\mathrm{SiO}_{2}$, in order to create lateral nucleation seeds for the Ge growth. The growth of $\mathrm{Ge}$ on Si surface close to the (111) orientation is of special interest, since in this orientation the misfit dislocations can glide along (111) planes. ${ }^{11}$ It is worth noting that the width of the seeds and the thickness of the $\mathrm{SiO}_{2}$ layer can be easily and precisely adjusted by controlling the thermal oxidation time and the etching time in HF solution. Figure 1(a) shows a cross sectional scanning electron microscopy (SEM) image of a linear silicon seed formed between $\mathrm{Si}_{3} \mathrm{~N}_{4}$ and $\mathrm{SiO}_{2}$. The width of the seed line is inferior to $50 \mathrm{~nm}$. The $\mathrm{SiO}_{2}$ layer thickness is around $50 \mathrm{~nm}$, and as expected, the surface orientation of the Si seed is close to (111).

The epitaxial growth of Ge on the so formed patterns is carried out in an ultrahigh vacuum chemical vapor deposition (UHV CVD) reactor with a base pressure of 2 $\times 10^{-10}$ Torr using germane $\left(\mathrm{GeH}_{4}\right)$ diluted at $10 \%$ in $\mathrm{H}_{2}$. As growth of $\mathrm{Ge}$ from $\mathrm{GeH}_{4}$ is naturally selective on $\mathrm{Si}$ versus silicon oxide, the growth conditions are chosen to avoid polycrystalline $\mathrm{Ge}$ deposition on the nitride mask. The growth temperature is set up at $600{ }^{\circ} \mathrm{C}$ under a total pressure of $1 \times 10^{-2}$ Torr, while the growth time varies between 1 and $2 \mathrm{~h}$.

The growth process is monitored in situ by reflection high energy electron diffraction (RHEED). The RHEED diagrams recorded during growth show only spotty and streaky patterns, no polycrystalline signature (rings) being detected, which indicates clearly a monocrystalline growth. Figure 1 shows cross sectional SEM images illustrating different stages of Ge lateral growth from the Si seeds. The Ge growth is selective, it starts from the Si seeds, as shown on Fig. 1(b), and proceeds laterally by "wetting" the $\mathrm{SiO}_{2}$ layer following 

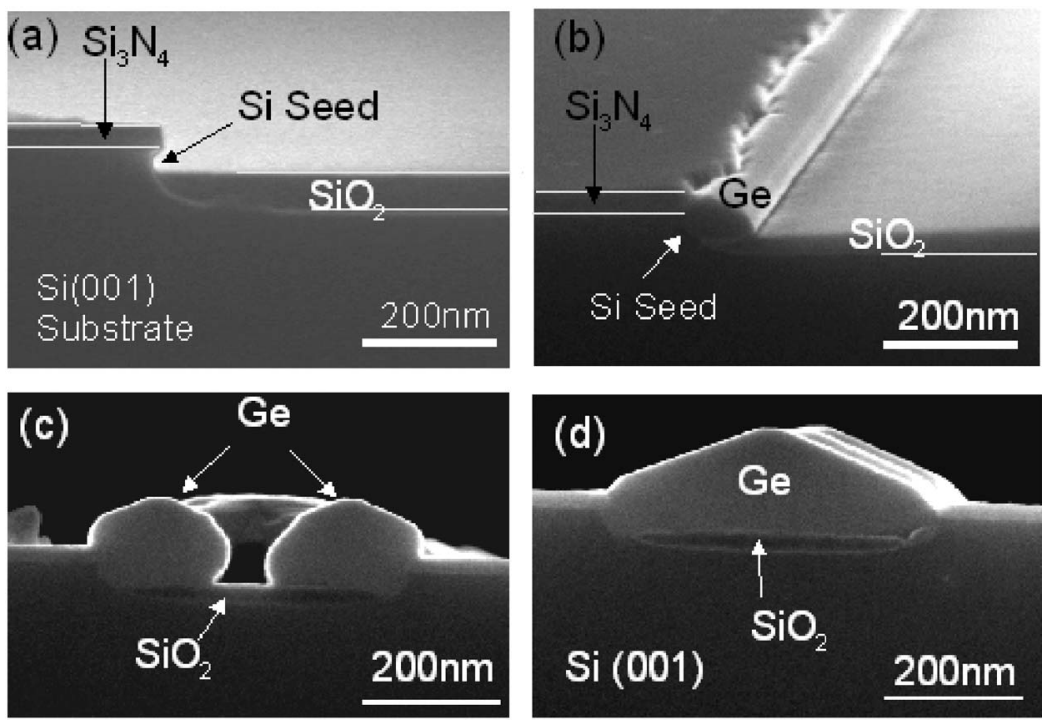

FIG. 1. Cross sectional SEM images of a linear Si seed oriented along the $\langle 110\rangle$ direction (a) and of Ge crystals grown from the $\mathrm{Si}$ seeds at different deposition times: $1 \mathrm{~h}$ (b), $1 \mathrm{~h}$ and $45 \mathrm{~min}$ (c), and $2 \mathrm{~h}$ (d). an angle close to $125^{\circ}$. This angle corresponds to $\{11-1\}$ facet, while the LEG Ge crystals exhibit $\{113\}$ and $\{111\}$ facets in their upper part [Fig. 1(c)]. Coalescence of Ge crystals from adjacent seeds takes place for longer deposition time and leads to rooflike structures delimited by $\{113\}$ and $\{111\}$ facets [Fig. 1(d)], as currently observed for selective epitaxial growth of Ge layers. ${ }^{12}$ The orientation of the seed patterns appears to be an essential parameter. The Ge crystals grows straight and parallel to the seed lines when these latter are oriented along the $\langle 110\rangle$ direction, as seen in Fig. 1(b). In the $\langle 110\rangle$ orientation, stable Ge facets can be formed. ${ }^{13}$ When seed lines are disoriented with respect to the $\langle 110\rangle$ direction, the Ge growth is more irregular, facet formation becomes unfavorable for lateral growth. This results in the formation of separated faceted crystals along the seeds, as shown in Fig. 2.

To better study the Ge structures, transmission electron microscopy (TEM) analysis is carried out on cross sectional specimens in a Cs-corrected Tecnai F20 installed at CEMESCNRS. As a first step, weak beam dark field analysis ${ }^{14}$ (WBDF) is used to investigate the presence of extended defects in the Ge crystals. Figure 3(a) is a WBDF image of a coalesced germanium crystal taken in a $g=220,(g,-2 g)$

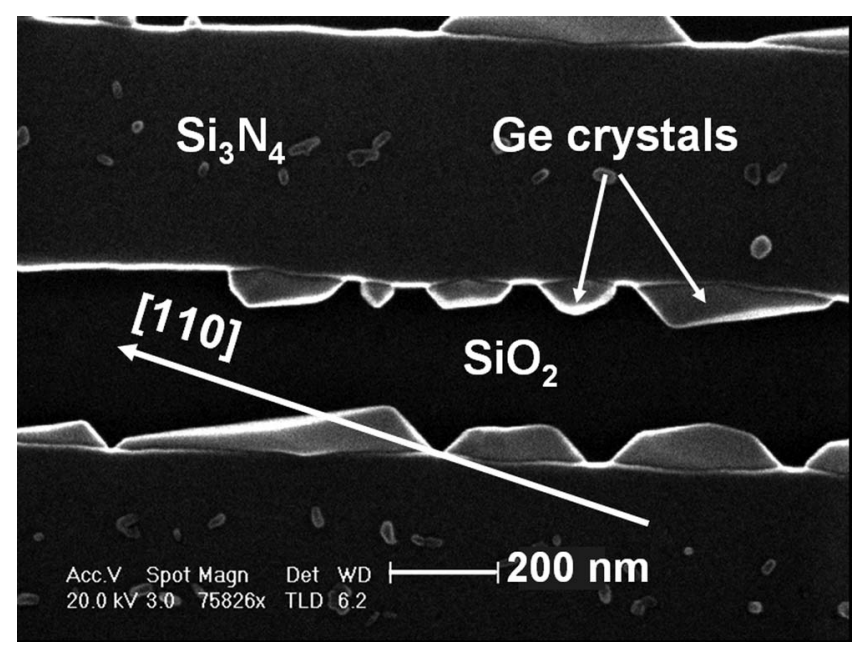

FIG. 2. Ge crystals grown from linear seeds disoriented by $22^{\circ}$ from the $\langle 110\rangle$ direction. condition. No defect is visible in the inner part of the structure while the contrast zones found at the two $\mathrm{Si} / \mathrm{Ge}$ interfaces confirm the presence of defects due to the misfit between bulk silicon and germanium. Moreover several plane view TEM analysis show that the joining of two front $\mathrm{s}$ result in defect-free interface. This means that the Ge crystals have evolved from a growth shape to an equilibrium shape during the coalescence, with a reorganization of the material that leads to a new grain-boundary-free Ge crystal.

These results are compatible with the hypothesis that LEG Ge consists in fully relaxed single crystals free of bulk defects. High Resolution TEM analysis (HRTEM) is carried out on the same specimen [Figs. 3(b) and 3(c)]. The recorded images confirm that as grown $\mathrm{Ge}$ is fully crystalline. In addition, HRTEM images obtained in the $\mathrm{Si} / \mathrm{Ge}$ interface region [Fig. 3(c)] indicate the presence of stacking fault s and
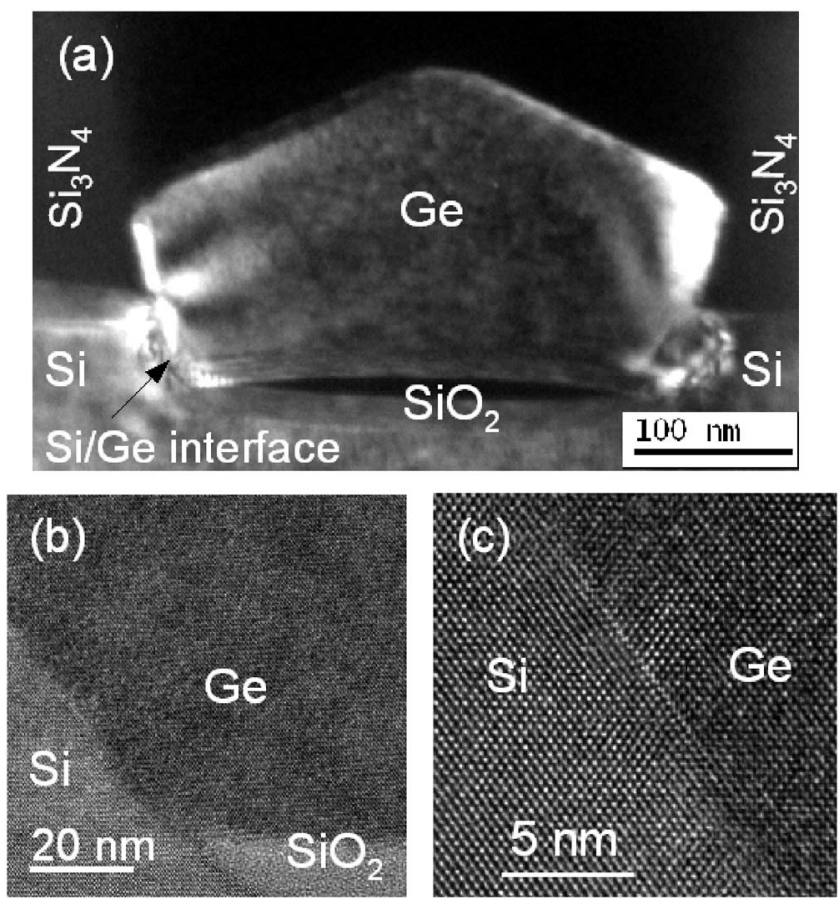

FIG. 3. TEM WBDF images obtained in a $g=220,(g,-2 \mathrm{~g})$ condition from a Ge crystal (a) and HREM images [(b) and (c)] of the Ge/Si interface. 


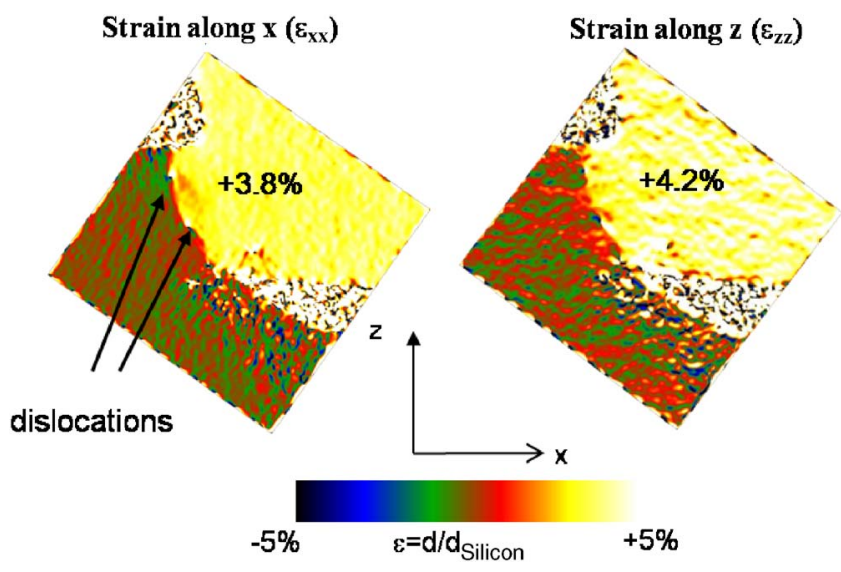

FIG. 4. (Color online) Two-dimensional strain maps of the Germanium seeds measured with reference to the silicon lattice. The strain value for relaxed germanium is $\varepsilon=4.2 \%$

interfacial dislocations at the interface. The defect density appears to be very low at the $\mathrm{Ge} / \mathrm{Si}$ interfaces. It is possible that the seed morphology (roughness and orientation) helps plastic relaxation. The roughness confines dislocations at interface, and, the (111) $\mathrm{Ge} / \mathrm{Si}$ interface enables misfit dislocations to glide in the interfacial plane. ${ }^{11}$

In order to evaluate the amount of strain in germanium crystals, geometric phase analysis of HRTEM images is performed. ${ }^{15,16}$ The lattice spacing is constant in the silicon layer far from the interface and it is used as the reference. As a consequence the measured strain $\varepsilon$ is a relative value with respect to an unstrained silicon lattice. In Fig. 4, the strain of the $(11-1)$ and $(200)$ planes are combined to determine the two-dimensional deformation tensor perpendicular to a $\langle 110\rangle$ zone axis. Two uniform zones of different constant strain are apparent in the image due to the change in the lattice spacing when passing from the silicon to the germanium region. It can be also observed, in Fig. 4, that the expansion of the $\mathrm{Ge}$ lattice with respect to relaxed Silicon is uniform in the (110) and (100) directions and amounts respectively to $\varepsilon_{x x}=$ $+3.8 \%$ and $\varepsilon_{z z}=+4.2 \%$. From these results we can conclude that the Ge crystal is almost fully relaxed, homogeneous and crystalline.

In conclusion, we have shown that large fully relaxed monocrystalline Ge crystals on $\mathrm{SiO}_{2}$ with very low defect densities can be easily obtained by LEG. A reproducible method based on LOCOS has been developed to create well localized nanoscale apertures through the $\mathrm{SiO}_{2}$ layer, that act as seeds for transferring the crystalline order from the $\mathrm{Si}$ substrate to the LEG Ge crystals. This local approach could be an attractive alternative to GeOI plain wafer for microand optoelectronic applications.

This work was supported by the Commission of European Communities IP Pullnano (IST contract No. 02828). The authors also thank the "Centrale de Technologie Universitaire" MINERVE for the technological backup.

${ }^{1}$ F. Letertre, C. Deguet, C. Richtarch, B. Faure, J. M. Hartmann, F. Chieu, A. Beaumont, J. Dechamp, C. Morales, F. Allibert, P. Perreau, S. Pocas, S. Personnic, C. Lagahe-Blanchard, B. Ghyselen, Y. M. Le Vaillant, E. Jalaguier, N. Kernevez, and C. Mazure, Proceedings of Symposium B of the MRS 2004 Spring Meeting, 12-16 April 2004, San Francisco, 2004 (unpublished).

${ }^{2}$ T. Akatsu, C. Deguet, L. Sanchez, F. Allibert, D. Rouchon, T. Signamarcheix, C. Richtarch, A. Boussagol, V. Loup, F. Mazen, J.-M. Hartmann, Y. Campidelli, L. Clavelier, F. Letertre, N. Kernevez, and C. Mazure, Materials Science in Semiconductor Processing 9, 444 (2006).

${ }^{3}$ T. Tezuka, N. Sugiyama, and S. Takagi, Appl. Phys. Lett. 79, 1798 (2001).

${ }^{4}$ D. Bouchier, V. Yam, D. Cammilleri, C. Renard, and F. Fossard, French Patent No. FR 2007/59859 (14 December 2007).

${ }^{5}$ F. R. N. Nabarro, Theory of Crystal Dislocations (Oxford, New York, 1967).

${ }^{6}$ R. People and J. C. Bean, Appl. Phys. Lett. 47, 322 (1985).

${ }^{7}$ S. Luryi and E. Suhir, Appl. Phys. Lett. 49, 140 (1986).

${ }^{8}$ Q. Li, B. Pattada, S. R. J. Brueck, S. Hersee, and S. M. Han, J. Appl. Phys. 98, 073504 (2005).

${ }^{9}$ M. Halbwax, C. Renard, D. Cammilleri, V. Yam, F. Fossard, D. Bouchier, Y. Zheng, and E. Rzepka, J. Cryst. Growth 308, 26 (2007).

${ }^{10}$ M. Okihara, S. Kuroda, M. Itoh, and N. Hirashita, Jpn. J. Appl. Phys., Part 1 34, 1822 (1995).

${ }^{11}$ Th. Schmidt, R. Kröger, J. I. Flege, T. Clausen, J. Falta, A. Janzen, P. Zal, P. Kury, M. Kammler, and H.-v. Hoegen, Phys. Rev. Lett. 96, 066101 (2006).

${ }^{12}$ M. Halbwax, L. H. Nguyen, F. Fossard, X. Le Roux, V. Mathet, V. Yam, D. T. Cao, and D. Bouchier, Mater. Sci. Semicond. Process. 9, 460 (2006).

${ }^{13}$ Z. Gai, W. S. Yang, R. G. Zhao, and T. Sakurai, Phys. Rev. B 59, 15230 (1999).

${ }^{14}$ D. B. Williams and C. B. Carter, Transmission Electron Microscopy A Textbook for Materials Science (Springer, New York, 2004).

${ }^{15}$ M. J. Hÿtch, E. Snoeck, and R. Kilaas, Ultramicroscopy 74, 131 (1998).

${ }^{16}$ M. J. Hÿtch, J. L. Putaux, and J. M. Pénisson, Nature (London) 423, 270 (2003). 